\title{
Heroin with a twist: candida glabrata endocarditis concurrent with brain mycetomas in an iv drug user
}

\begin{abstract}
A 47-year-old male, with a history of IV drug use and usage of fresh lemon juice in the preparation of heroin doses, with hepatitis $\mathrm{B}$ and hepatitis $\mathrm{C}$, presented with fever, dyspnea and a new found cardiac murmur at the apex. Echocardiography showed large vegetations on both leaflets of the mitral valve. After blood cultures were drawn an empiric antibiotic regimen was initiated. The mitral valve was successfully replaced surgically. Twenty-four hours post surgically the patient was extubated and was found to be suffering from left hemiplegia. Brain CT scan showed diffused sites of hemorrhage of the brain parenchyma, compatible with septic emboli that had underwent a hemorrhagic conversion. As mycetomas were considered in the differential diagnosis of these lesions, new blood cultures were drawn with an emphasis on the isolation of fungi, as this correlates with the use of lemon juice in the preparation of heroin doses, while the treatment regimen was modified to include an echinocandin to cover extensively for Candida spp. Two sets of blood cultures were positive for Candida glabrata. The patient responded well to the treatment administered and regained a significant amount of mobility.
\end{abstract}

Keywords: candida glabrata, endocarditis, mycetomas, iv drug use, mitral valve replacement
Volume 5 Issue 3 - 2017

\author{
Loukas Kakoullis,' Marina Andreou,' Antreas \\ loannou, ${ }^{2}$ Dionysios CWatson, ${ }^{1,3}$ Eleni \\ Papachristodoulou,' Paraskevi Chra, ${ }^{4}$ George \\ Panos ${ }^{1,2}$ \\ 'Department of Internal Medicine, University of Patras, Greece \\ ${ }^{2}$ Department of Internal Medicine, University of Cyprus Medical \\ School, Cyprus \\ 3Center for Cancer research, National Institute of Health, USA \\ ${ }^{4}$ Department of Microbiology, Korgialenio Benakio Red Cross \\ Hospital, Greece
}

\begin{abstract}
Correspondence: George Panos MD, FRCP, Department of Internal Medicine, Division of Infectious Diseases, University of Patras, School of Medicine, Patras, Greece \& Department of Internal Medicine, Nicosia General Hospital, University of Cyprus Medical School, Cyprus , Tel 35722603447, Fax 35722604322,Email George.panos.frcp@gmail.com
\end{abstract}

Received: May 15, 2017| Published: July 20, 2017

Abbreviations: ICU, intensive care unit; CT, computed tomography; VKA, vitamin k antagonist; LMWH, low molecular weight heparin; BUN, blood urea nitrogen; INR, international normalized ratio; IV, intravenous; IVDUs, intravenous drug users; IDSA, infectious disease society of America

\section{Introduction}

Fungal endocarditis is quite uncommon, representing only $2 \%$ among cases of infective endocarditis. ${ }^{1}$ Risk factors include the presence of prosthetic valves, short term indwelling catheters, broad spectrum antibiotic use, history of health associated care and intravenous drug use. ${ }^{1,2}$ A distinct clinical syndrome of disseminated candidiasis in heroin abusers exists ${ }^{3}$ and it is associated with the use of lemon in the preparation of heroin prior to injection. ${ }^{4}$ Manifestations include development of lesions in the skin of hair bearing areas such as the scalp, the beard, and the axillary and pubic areas where pustules are formed in the location of follicles, ocular lesions such as episcleritis and chorioretinitis, and lesions in bone and costal cartilage. ${ }^{3}$ Candida endocarditis complicates candidaemia in up to $17.7 \%$ of patients ${ }^{5}$ and is associated with a mortality rate of $30.3 \%$, nearly double of that of bacterial infective endocarditis. ${ }^{2}$

The frequency of isolation of non-albicans Candida spp. is increasing ${ }^{6}$ and Candida glabrata is of special interest, as it is intermittently resistant to all azoles and, even if sensitive, can subsequently develop secondary resistance during therapy with fluconazole in $20 \%$ of cases. Azole resistance results from point mutations to ERG11, the gene of the target enzyme of azoles. However C. glabrata remains susceptible to Voriconazole, polyenes and echinocandins. ${ }^{7}$ Nevertheless, resistance to these antifungals is emerging. An acquired mutation to fks1 or fks2 genes in fluconazole resistant isolates is associated with resistance to echinocandins. Of all fluconazole resistant strains, $11.1 \%$ are resistant to one or more echinocandins, and all of these carry a mutation in one of these genes. The emergence of this co-resistance reflects the selection pressure exerted on this organism, as echinocandins are administered to immunocompromised patients with prior exposure to azoles. ${ }^{8}$ Resistance to polyenes has been reported and has been attributed to defective ergosterol biosynthesis. ${ }^{7}$

\section{Case presentation}

A 47 year old male with past medical history of chronic IV drug abuse and chronic hepatitis $\mathrm{B}$ and $\mathrm{C}$ coinfection, was referred from another hospital because of symptomatic mitral insufficiency. The patient complained of fever and progressively worsening dyspnea, beginning a month prior to his admission. During physical examination, the patient was febrile (fever of $38.5 \mathrm{oC}$ ), tachypneic, with a systolic murmur at the apex. Laboratory workup showed norm chromic anemia and a subtle rise in BUN and creatinine, in line with the presence of heart failure. A transthoracic echocardiogram revealed the presence of large vegetations on the posterior and anterior leaflet of the mitral valve with severe mitral regurgitation and mild pulmonary hypertension. The diagnosis of infective endocarditis was made and, after initial blood cultures were drawn, empiric treatment regimen was initiated with intravenous Meropenem, Daptomycin, Fluconazole and Rifampicin.

The patient urgently underwent successful replacement of the mitral valve immediately after admission where two large vegetations were removed from the anterior and posterior leaflet (measuring two centimeters each). Culture and histopathology of the vegetations were negative. After being intubated for 24 hours in ICU, the patient was extubated and left hemiplegia was noted with findings of septic emboli with hemorrhagic transformation on urgent CT Brain scan. Additional imaging studies, revealed septic infarcts in both the spleen and kidneys.

An infectious disease specialist was consulted and four blood cultures were drawn with emphasis for isolating fungi, since brain parenchyma lesions could be clinically attributed to mycetomas. The 
patient when asked directly, admitted to using fresh lemon juice in the heroin preparation. The new sets of blood cultures were positive for C. glabrata, resistant to all azoles (only intermediate sensitivity to Posaconazole) and sensitive to amphotericin-B and all echinocandins. Re-evaluation of the histopathology sample for fungi, with PAS staining, revealed brown spongiform projections, compatible with fungi.

Since the patient was still febrile a week after administrating the initial antimicrobial regimen, the treatment was switched to Vancomycin, Micafungin and Aztreonam (due to local resistance patterns to Pseudomonas spp.) while Rifampicin was continued. Due to pharmacy shortage, Micafungin was switched to Caspofungin. In addition anticoagulation treatment with a vitamin $\mathrm{K}$ antagonist (VKA) was initiated and subsequently switched to low molecular weight heparin (LMWH) due to drug interaction between Rifampicin and VKA which led to difficulty in controlling INR.

The patient gradually improved neurologically with improvement of his left lower limb weakness and size reduction of the brain haemorrhagic infarcts, on follow-up CT Brain scans. The patient however re-presented fever and from repeated blood cultures Enterococcus faecium was isolated and Linezolid was added to the antimicrobial regimen, with his fever subsiding two days after. The patient was relocated to another hospital near his home, two months after his admission. There he continued his antifungal treatment, showing clinical improvement, while being a febrile with partial recovery of his left hemiparesis. He was discharged after completing a total of 12 weeks of echinocandin treatment.

During follow-up examination at our Outpatient Department the patient was improved with residual left hemiparesis. Treatment with oral Posaconazole was administered for six more months on an outpatient basis, with clinical improvement of left hemiparesis and improvement of brain residual mycetomas. Complete regression of splenic and renal infarcts was noted. Due to minimal CNS penetration by Posaconazole, administration of Liposomal amphotericin B was considered for the elimination residual brain mycetomas.

\section{Discussion}

Fungi comprise less than $2 \%$ of all cases of infective endocarditis ${ }^{1,2,9}$ with Candida spp. representing 50-80\% of them and C. albicans being the most commonly isolated species (30-40\%). Non-albicans Candida spp. endocarditis (C. parapsilosis, C. glabrata, C. tropicalis) show an increasing trend, ${ }^{5}$ due to previous exposure to azoles. ${ }^{1}$ Aspergillus spp. represents $20-25 \%$ of fungal endocarditis cases, especially in patients with severe immunosuppression, prosthetic valves or hematological malignancies. Among Aspergillus spp., two thirds of the cases are attributed to A. fumigatus. ${ }^{1}$

Predisposing risk factors for fungal endocarditis include recent healthcare exposure, use of broad spectrum antibiotics, use of shortterm indwelling catheters, prosthetic heart valves and intravenous drug users (IVDUs). ${ }^{1,2,5}$ Higher mortality rates have been noted in these cases ${ }^{2}$, with development of large vegetations and subsequent increased risk for septic emboli and unique skin lesions (according to the fungi isolated). Their clinical characteristics are similar to bacterial infective endocarditis and that is why modified Duke criteria still apply for its diagnostic approach.

Blood culture remains the mainstay of Candida endocarditis diagnosis (with compatible findings in transesophageal echocardiography), despite the fact that are yielded positive only in $50-75 \%$ of cases in C. albicans and less often in non-albicans species. ${ }^{1,10}$ Fungemia might be suspected also from spurious increase of white blood cell count or platelet count from a complete blood count. ${ }^{11}$ These findings are explained by the fact that automated blood analyzers may misidentify Candida spp. as platelets or white blood cells depending on the size and light scattering capabilities of different Candida species. ${ }^{12,13}$

The color of $C$. glabrata isolate has been associated with virulence. The fungus can reversibly switch between white, light brown and dark brown phenotypes, with the dark brown phenotype being the most virulent and the commonly isolated from clinical samples. Compared to the white phenotype, the dark brown phenotype colonizes the spleen and liver more efficiently and maintains its presence in these organs, as well as the kidney, for longer, whereas the white phenotype has already been cleared. ${ }^{14}$ In this case the isolate was brown; however, the pathology report did not specify whether it was of the light or dark phenotype.

Spleen infarction is a rare complication of infective endocarditis and will evolve to a spleenic abscess in $5 \%$ of patients. The formation of a spleenic abscess is associated with an increased morbidity and mortality, unless treatment is administered promptly..$^{15}$ It is unknown whether the development of this complication contributes to the overall immunosuppression, often present in patients with infective endocarditis. Although medical treatment alone is usually insufficient in cases of bacterial etiology, fungal spleenic abscesses can be managed adequately by antifungals alone. ${ }^{16}$ As stated above, the brown phenotype of C. glabrata shows an increased tropism for the spleen, compared to the white phenotype. ${ }^{14}$

Use of lemon juice as a heroin solvent and its implication in disseminated candidiasis was first identified in Australia in $1983^{17,18}$ and a relevant distinct clinical syndrome was described. ${ }^{3}$ Research demonstrated that lemon juice can support $C$. albicans growth at room temperature ${ }^{19}$ and was associated with disseminated candidiasis or Candida endophthalmitis in IVDUs, since lemon juice was used for preparation of brown heroin for intravenous use. ${ }^{4,20-22}$

According to Infectious Disease Society of America (IDSA) guidelines of $2016,{ }^{23}$ initial therapy for native valve Candida spp. endocarditis consists of either high dose echinocandin (Caspofungin $150 \mathrm{mg}$ daily, Micafungin 150mg daily, or Anidulafungin $200 \mathrm{mg}$ daily) or lipid formulation Amphotericin B, 3-5mg/ $\mathrm{kg}$ daily, with or without IV Flucytosine, $25 \mathrm{mg} / \mathrm{kg} 4$ times daily. Step down therapy with fluconazole for patients with susceptible Candida isolates is recommended, provided they have demonstrated clinical stability and have cleared the fungus from the bloodstream. If the isolate is resistant to fluconazole but susceptible to Voriconazole or Posaconazole, then these agents can be used instead. The same regimens are recommended for the treatment of prosthetic valve endocarditis, with the addition of chronic suppressive antifungal therapy with fluconazole, to prevent recurrence.

Valve replacement is recommended, with treatment continued for at least 6 weeks after surgery and for a longer duration in patients with perivalvular abscesses and other complications. For patients who cannot undergo valve replacement, long-term suppression with fluconazole, $400-800 \mathrm{mg}(6-12 \mathrm{mg} / \mathrm{kg})$ daily, if the isolate is susceptible, is recommended.

Anticoagulation therapy is also indicated in patients that receive prosthetic heart valves with VKAs being the mainstay of anticoagulation regimen. However, LMWH have been proposed as a safe alternative, in case drug interactions are present, due to cytochrome $\mathrm{p} 450$ inhibition or induction, such in this case. ${ }^{24,25}$ 


\section{Acknowledgements}

None.

\section{Conflicts of interest}

The authors report no conflict of interest.

\section{Funding}

None.

\section{References}

1. Tattevin P, Revest M, Lefort A, et al. Fungal endocarditis: current challenges. Int J Antimicrob Agents. 2014;44(4):290-294.

2. Baddley JW, Benjamin DK, Patel M, et al. Candida Infective Endocarditis. Eur J Clin Microbiol Infect Dis. 2008;27(7):519-529.

3. Collignon PJ, Sorrel TC. Disseminated candidiasis: evidence of a distinctive syndrome in heroin abusers. Br Med J (Clin Res Ed). 1983;287(6396):861-862.

4. Bisbe J, Miro JM, Latorre X, et al. Disseminated candidiasis in addicts who use brown heroin: report of 83 cases and review. Clin Infect Dis. 1992;15(6):910-923.

5. Lefort A, Chartier B, Sendid M, et al. Diagnosis, management and outcome of Candida endocarditis. Clin Microbiol Infect. 2012;18(4):E99-E109.

6. Fidel PL, Vazquez JA, Sobel JD. Candida glabrata: review of epidemiology, pathogenesis, and clinical disease with comparison to $C$. albicans. Clin Microbiol Rev. 1999;12(1):80-96.

7. Silva S, Negri M, Henriques M, et al. Candida glabrata, Candida parapsilosis and Candida tropicalis: biology, epidemiology, pathogenicity and antifungal resistance. FEMS Microbiol Rev. 2012;36(2):288-305.

8. Katiyar SK, Alastruey Izquierdo A, Healey KR, et al. Fks1 and Fks2 are functionally redundant but differentially regulated in Candida glabrata: implications for echinocandin resistance. Antimicrob agents chemother. 2012;56(12):6304-6309.

9. Arnold CJ, Johnson M, Bayer AS, et al. Candida infective endocarditis: an observational cohort study with a focus on therapy. Antimicrob Agents Chemother. 2015;59(4):2365-2373.

10. Cornely OA, Bassetti M, Calandra T, et al. ESCMID guideline for the diagnosis and management of Candida diseases 2012: non-neutropenic adult patients. Clin Microbiol Infect. 2012;18(supp 7): 19-37.
11. Latif S, Veillon DM, Brown D, et al. Spurious Automated Platelet Count. Enumeration of yeast forms as platelets by the cell-DYN 4000 . Am J Clin Pathol. 2003;120(6):882-885.

12. Kim HR, Park BR, Lee MK. Effects of bacteria and yeast on WBC counting in three automated hematology counters. Ann Hematol. 2008;87(7):557-562

13. Branda JA, Kratz A. Effects of yeast on automated cell counting. Am J Clin Pathol. 2006;126(2):248-254.

14. Srikantha T, Daniels KJ, Wu W, et al. Dark brown is the more virulent of the switch phenotypes of Candida glabrata. Microbiology. 2008;154(11):3309-3318.

15. Elasfar A, Al Baradai A, AlHarfi Z, et al. Splenic abscess associated with infective endocarditis; Case series. J Saudi Heart Assoc. 2015;27(3):210-215

16. Farres H, Felsher J, Banbury M, et al. Management of splenic abscess in a critically ill patient. Surg Laparosc Endosc Percutan Techn. 2004;14(2):49-52.

17. Dally S, Thomas G, Danan M. Candidiasis in heroin abusers. Br Med J (Clin Res Ed). 1983; 287(6404):1549.

18. Mackay M. Candidiasis in heroin abusers. Br Med J (Clin Res Ed). 1983;287(6408):1885.

19. Newton John HF, Wise K, Looke DFM. Role of the lemon in disseminated candidiasis of heroin abusers. Med J Aust. 1984;140(13):780-781.

20. Odds FC, Palacio Hernanz AM, Cuadra J, et al. Disseminated Candida infection syndrome in heroin addictsominance of a single Candida albicans biotype. J Med Microbiol. 1987;23(3):275-277.

21. Strang J, Keaney F, Butterworth G, et al.Different forms of heroin and their relationship to cook-up techniques: data on, and explanation of, use of lemon juice and other acids. Subst Use Misuse. 2001;36(5):573-588.

22. Albini TA, Sun RL, Holz ER, et al. Lemon juice and Candida endophthalmitis in crack-cocaine misuse. $\mathrm{Br} \quad J$ Ophthalmol. 2007;91(5):702-703.

23. Pappas PG, Kauffman CA, Andes DR, et al. Clinical practice guideline for the management of candidiasis: 2016 update by the Infectious Diseases Society of America. Clin Infect Dis. 2015; 62(4):e1-50.

24. Fahmi AM, Abdelsamad O, Elewa H. Rifampin-warfarin interaction in a mitral valve replacement patient receiving rifampin for infective endocarditis: a case report. SpringerPlus. 2016;5(1):8.

25. Montalescot G, Polle V, Collet JP, et al. Low molecular weight heparin after mechanical heart valve replacement. Circulation. 2000;101(10):1083-1086. 